\title{
A BEM BASED ON THE BÉZIER/BERNSTEIN POLYNOMIAL FOR ACOUSTIC WAVEGUIDE MODELIZATION
}

\author{
ANTONIO ROMERO ${ }^{1}$, PEDRO GALVÍN ${ }^{1} \&$ ANTÓNIO TADEU ${ }^{2,3}$ \\ ${ }^{1}$ Escuela Técnica Superior de Ingeniería, Universidad de Sevilla, Spain \\ ${ }^{2}$ ITeCons - Institute for Research and Technological Development in Construction, Energy, \\ Environment and Sustainability, Portugal \\ ${ }^{3}$ ADAI - LAETA, Department of Civil Engineering, University of Coimbra, Portugal
}

\begin{abstract}
This paper proposes a novel boundary element approach formulated on the Bézier-Bernstein basis to yield a geometry-independent field approximation. The proposed method is geometrically based on both computer aided design (CAD) and isogeometric analysis (IGA), but field variables are independently approximated from the geometry. This approach allows the appropriate approximation functions for the geometry and variable field to be chosen. We use the Bézier-Bernstein form of a polynomial as an approximation basis to represent both geometry and field variables. The solution of the element interpolation problem in the Bézier-Bernstein space defines generalised Lagrange interpolation functions that are used as element shape functions. The resulting Bernstein-Vandermonde matrix related to the Bézier-Bernstein interpolation problem is inverted using the Newton-Bernstein algorithm. The applicability of the proposed method is demonstrated by solving the Helmholtz equation over an unbounded region in a two-and-a-half dimensional (2.5D) domain.
\end{abstract}

Keywords: subparametric method, Bézier-Bernstein curve, Newton-Bernstein algorithm, computeraided design, isogeometric analysis.

\section{INTRODUCTION}

We present a geometry-independent field approximation of the boundary element method (BEM). The proposed formulation shares some characteristics with the standard collocation methods and isogeometric analysis (IGA), exploring the advantages of each method. A similar concept was recently introduced by Atroshchenko and Bordas [1].

The geometry is represented by Bézier curves defined as a linear combination of Bernstein basis with control points. The Bernstein basis is widely used in CAD because of its good properties for building and modifying geometric shapes [2]. On the other hand, the use of orthogonal basis allows simple and efficient field approximation. To define an orthogonal basis for the field interpolation, therefore, a least-square problem in terms of BernsteinVandermonde matrix is generated by the interpolation constraints whose solution is a Lagrange interpolant relative to the Bernstein basis [3], [4]. This methodology is an efficient and robust approach that combines the Bernstein basis for geometry representation and the Lagrange interpolating polynomial relative to the Bernstein basis for the field approximation.

The proposed method is powerful because it includes the main characteristics of standard BEM formulation and isogeometric analysis: (i) the geometry is exactly defined as Bézier curves; (ii) field variables are represented at nodal points instead of control variables, which enables easy definition of the boundary conditions; (iii) $h$-refinement is performed directly on the geometry without CAD interaction, as it is performed by IGA; and (iv) $p$-refinement only affects the field approximation, rather than the geometry discretisation.

In this paper, the capability of the proposed method is demonstrated for solving the Helmholtz equation in longitudinally invariant problems. This situation is referred to as a twoand-a-half dimensional (2.5D) problem [5], in which the three-dimensional (3D) solution is 
computed by means of a Fourier transform. The structure of the rest of the paper is as follows. First, the Bézier-Bernstein space is briefly described and the Lagrange interpolant relative to the Bernstein basis is derived. Next, the boundary element formulation in the BézierBernstein space is presented, after which, the proposed methodology is verified by means of two benchmark problems and a numerical example is presented.

\section{NUMERICAL MODEL}

This approach is based on the Helmholtz integral equation for solving 3D interior, exterior and scattering problems in the frequency-wavenumber domain. The pressure field for the acoustic waveguide at point $\mathbf{x}=\mathbf{x}(x, y, z)$ is computed as the superposition of 2D solutions with different wavenumbers $\kappa_{z}[6]$ :

$$
p(\mathbf{x}, \omega)=\int_{-\infty}^{+\infty} \widetilde{p}\left(\widetilde{\mathbf{x}}, \kappa_{z}, \omega\right) e^{-\iota \kappa_{z} z} d \kappa_{z}
$$

where $\widetilde{p}\left(\widetilde{\mathbf{x}}, \kappa_{z}, \omega\right)$ is the frequency-wavenumber representation of the sound pressure, $\widetilde{\mathbf{x}}=$ $\mathbf{x}(x, y)$ and $\iota=\sqrt{-1}$.

The boundary integral representation is derived from the Helmholtz equation. The fundamental solution $\widetilde{\Psi}\left(\widetilde{\mathbf{x}}, \kappa_{z}, \omega ; \widetilde{\mathbf{x}}_{i}\right)$ for sound pressure at receiver position $\widetilde{\mathbf{x}}$ due to a source acting at $\widetilde{\mathbf{x}}_{i}$ is the solution to:

$$
\nabla^{2} \widetilde{\Psi}\left(\widetilde{\mathbf{x}}, \kappa_{z}, \omega ; \widetilde{\mathbf{x}}_{i}\right)+\kappa^{2} \widetilde{\Psi}\left(\widetilde{\mathbf{x}}, \kappa_{z}, \omega ; \widetilde{\mathbf{x}}_{i}\right)=-\delta(r),
$$

where $\kappa=\omega / c_{f}$ is the problem wavenumber, $c_{f}$ is the sound propagation velocity, $\delta$ is the Dirac delta function and $r=\left\|\widetilde{\mathbf{x}}_{i}-\widetilde{\mathbf{x}}\right\|$ is the distance from the source to the receiver. The solution to this equation defines the fundamental solution for an unbounded region in the frequency-wavenumber:

$$
\widetilde{\Psi}\left(\widetilde{\mathbf{x}}, \kappa_{z}, \omega ; \widetilde{\mathbf{x}}_{i}\right)=-\frac{\iota}{4} H_{0}^{(2)}\left(\kappa_{\alpha} r\right),
$$

where $\kappa_{\alpha}=\sqrt{\kappa^{2}-\kappa_{z}^{2}}$ and $H_{0}^{(2)}$ is the Hankel function.

The following sections deal with: (i) the Bézier-Bernstein space for geometry representation, (ii) the Lagrange interpolant relative to Bernstein basis for variable field approximation, and (iii) the boundary element formulation in the Bézier-Bernstein space.

\subsection{The Bézier-Bernstein space}

The Bernstein basis of degree $n$ is defined over the interval $t \in[0,1]$ as:

$$
B_{k}^{n}(t)=\left(\begin{array}{l}
n \\
k
\end{array}\right) t^{k}(1-t)^{n-k}, \quad k=0, \ldots, n .
$$

The change of variable to map $x \in[a, b]$ to $t \in[0,1]$ allows the definition of the Bernstein polynomial related to a continuous function $f(t)$ as follows:

$$
P_{n}(t)=\sum_{k=0}^{n} f\left(\frac{k}{n}\right) B_{k}^{n}(t) .
$$

Then, eqn (5) converges to $f(t)$ as $n$ goes to infinity due to the Bernstein basis being nonnegative and forming a partition unity, although the polynomial form does not interpolate 
the sample values $f(k / n)$. The Bernstein basis has partition unity property, is defined as non-negative and symmetric about the interval mid-point $\left(B_{n-k}^{n}(1-t)=B_{n}^{k}(t)\right)$.

The application of polynomials in Bernstein form grows with the development of Bézier curves $\mathbf{r}_{n}(t)$ in computer-aided design:

$$
\mathbf{r}_{n}(t)=\sum_{k=0}^{n} \mathbf{b}_{k} B_{k}^{n}(t)
$$

where $\mathbf{b}_{k}$ are the control points used to approximate the geometry. A control polygon is obtained by connecting the control points and this is used to modify the curve geometry. The de Casteljau algorithm is often used for evaluating and splitting a Bézier curve $\mathbf{r}_{n}(t)$ at a given point $t$ [2].

Although the de Casteljau algorithm allows an easy evaluation of a Bézier curve, it is computationally expensive. An efficient curve computation is achieved using the polar form (or blossom) of a Bézier curve $\mathbf{r}_{n}(t)$ [7], which defines a multi-affine transformation satisfying:

$$
\mathbf{b}_{k}=\mathbf{R}(\underbrace{0, \ldots, 0}_{n-k}, \underbrace{1, \ldots, 1}_{k})
$$

where $\mathbf{R}\left(t_{1}, \ldots, t_{n}\right)$ is computed as:

$$
\mathbf{R}\left(t_{1}, \ldots, t_{n}\right)=\sum_{\substack{I \cap J=\emptyset \\ I \cup J=\{1,2, \ldots, n\}}} \prod_{i \in I}\left(1-t_{i}\right) \prod_{j \in J} t_{j} \mathbf{b}_{|J|} \cdot
$$

Thus, a polynomial in Bernstein form can be formulated in polar form substituting eqn (7) into eqn (6) as follows:

$$
\mathbf{r}_{n}(t)=\sum_{k=0}^{n} \mathbf{R}(\underbrace{0, \ldots, 0}_{n-k}, \underbrace{1, \ldots, 1}_{k}) B_{k}^{n}(t)=\mathbf{R}(t, \ldots, t) .
$$

The polar form of a Bézier curve $\mathbf{r}_{n}(t)$ of degree $n$ defines a multi-affine polynomial $\mathbf{R}\left(t_{1}, \ldots, t_{n}\right)$ with $n$ variables that satisfy $\mathbf{r}_{n}(t)=\mathbf{R}(t, \ldots, t)$.

Cubic Bézier approximations are widely used to represent smooth patches in CAD. More complex shapes would be defined by either increasing the curve order or using a piecewise Bézier curve. Fig. 1 shows a third-order Bézier patch and its related control points represented in polar form.

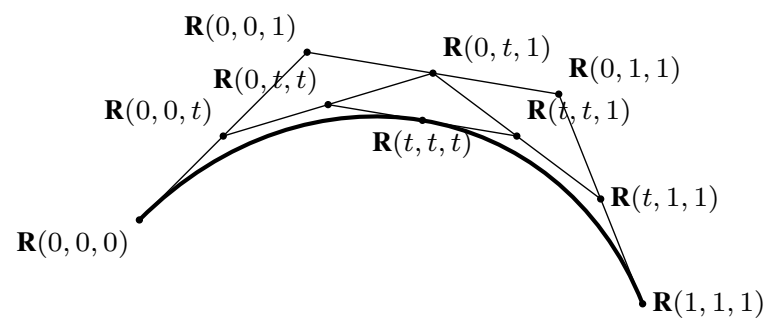

Figure 1: Third-order Bézier patch. 
The geometry approximation presented in this section is used to formulate the BEM in the Bézier-Bernstein space. The boundary geometry is defined by patches with an arbitrary approximation degree, that depends on the geometry shape complexity. Then, each patch is subdivided into elements according to $h$-refinement.

\subsection{Field approximation}

The Bézier-Bernstein space allows the approximation of a curve geometry through control points. Moreover, the element shape function can be derived from the Lagrange interpolant relative to the Bernstein basis presented in the previous section. The Lagrange interpolant relative to the Bernstein basis has become useful for high-order element development [12]. Although a polynomial in Bernstein form satisfies $P_{n}(0)=f(0)$ and $P_{n}(1)=f(1)$, these properties do not remain valid at internal element points as has been stated. Therefore, it is necessary to properly modify the control points $b_{k}$ to ensure the following condition:

$$
P_{n}\left(x_{i}\right)=\sum_{k=0}^{n} b_{k} B_{k}^{n}\left(x_{i}\right)=f\left(x_{i}\right) .
$$

This condition is commonly expressed as a linear system of equations through the BernsteinVandermonde matrix $A_{i j}=B_{i}^{j}\left(x_{i}\right)$, for $i, j=0, \ldots, n$. The control points $\mathbf{b}=\left[b_{0}, \ldots, b_{n}\right]^{T}$ are given by the solution of $\mathbf{A b}=\mathbf{f}$, where the vector $\mathbf{f}=\left[f\left(x_{0}\right), \ldots, f\left(x_{n}\right)\right]^{T}$ collects the interpolation data.

In this work, we have used the Newton-Bernstein algorithm proposed by Ainsworth and Sánchez [11] to compute the control points. The Newton-Bernstein algorithm [11] starts with the Newton form of the interpolant $P_{n}$ at nodes $x_{i}$ :

$$
P_{k}(x)=\sum_{i=0}^{k} f\left[x_{0}, \ldots, x_{k}\right] w_{i}(x), \quad k=0, \ldots, n,
$$

where $f\left[x_{0}, \ldots, x_{k}\right]$ is the divided difference defined by the following recursive formula:

$$
f\left[x_{j}, \ldots, x_{k}\right]=\frac{f\left[x_{j+1}, \ldots, x_{k}\right]-f\left[x_{j}, \ldots, x_{k-1}\right]}{x_{k}-x_{j}}, \quad k=j+1, \ldots, n ; \quad j=0, \ldots, n .
$$

Thus, the following expressions for control points $w_{k}$ and $b_{k}$, for $k=0, \ldots, n$ are defined:

$$
\begin{aligned}
& w_{k}\left(x_{j}\right)=\frac{j}{k} w_{k-1}\left(x_{j-1}\right)\left(1-x_{k-1}\right)-\frac{k-j}{k} w_{k-1}\left(x_{j}\right) x_{k-1}, \\
& b_{k}\left(x_{j}\right)=\frac{j}{k} b_{k-1}\left(x_{j-1}\right)+\frac{k-j}{k} b_{k-1}\left(x_{j}\right)+w_{k}\left(x_{j}\right) f\left[x_{0}, \ldots, x_{k}\right],
\end{aligned}
$$

and for $j=0, \ldots, k$ the latter expressions are $w_{0}\left(x_{0}\right)=1, b_{0}\left(x_{0}\right)=f\left[x_{0}\right], w_{-1}\left(x_{k-1}\right)=$ $b_{-1}\left(x_{k-1}\right)=0$ and $w_{k}\left(x_{k-1}\right)=b_{k}\left(x_{k-1}\right)=0$.

The Lagrange polynomial $P_{n}$ of order $n$ defined through the Bernstein basis gives $n+1$ interpolation functions $\phi^{j}$ computed after imposing the constrains $P_{n}^{j}\left(x_{k}\right)=\phi^{j}\left(x_{k}\right)=$ $\delta\left(x_{k}-x_{j}\right)$, for $j, k=0, \ldots, n$. Then, $\mathbf{b}$ becomes a matrix of unknown control points and $\mathbf{f}=\mathbf{I}$ is the identity matrix. The related interpolation functions are computed for a given point $x$ as $\left[\phi^{1}(x), \ldots, \phi^{j}(x), \ldots, \phi^{n+1}(x)\right]^{T}=\mathbf{b A}(x)$. The evaluation of the shape functions also benefits from the computational advantages of using the polar form given in the previous section. 


\subsection{Boundary element formulation}

Since the Bézier-Bernstein basis allowed the independent geometry and field variable approximation, the formulation of the BEM in Bézier-Bernstein space is quite straightforward, as in a classical formulation. This section describes the boundary element formulation for potential problems in acoustics [13]. The integral representation of the sound pressure in the frequency-wavenumber domain for a point $\widetilde{\mathbf{x}}_{i}$ located at the arbitrary boundary $\Gamma$ can be written as:

$$
\begin{aligned}
& c_{i}\left(\widetilde{\mathbf{x}}_{i}\right) \widetilde{p}_{i}\left(\widetilde{\mathbf{x}}_{i}, \kappa_{z}, \omega\right) \\
& \quad=-\int_{\Sigma}\left(\iota \rho \omega \widetilde{v}_{i}\left(\widetilde{\mathbf{x}}_{i}, \kappa_{z}, \omega\right) \widetilde{\Psi}\left(\widetilde{\mathbf{x}}, \kappa_{z}, \omega ; \widetilde{\mathbf{x}}_{i}\right)+\widetilde{p}_{i}\left(\widetilde{\mathbf{x}}_{i}, \kappa_{z}, \omega\right) \frac{\partial \widetilde{\Psi}\left(\widetilde{\mathbf{x}}, \kappa_{z}, \omega ; \widetilde{\mathbf{x}}_{i}\right)}{\partial \mathbf{n}}\right) d \Sigma,
\end{aligned}
$$

where $\widetilde{p}\left(\widetilde{\mathbf{x}}_{i}, \kappa_{z}, \omega\right)$ and $\widetilde{v}\left(\widetilde{\mathbf{x}}_{i}, \kappa_{z}, \omega\right)$ are the sound pressure and the particle normal velocity at the cross section $\Sigma$ of the boundary $\Gamma$, respectively. $\widetilde{\Psi}\left(\widetilde{\mathbf{x}}, \kappa_{z}, \omega ; \widetilde{\mathbf{x}}_{i}\right)$ is the solution to the Helmholtz equation at point $\widetilde{\mathbf{x}}$ due to a point source located at $\widetilde{\mathbf{x}}_{i}$ (eqn (3)). The integral-free term $c_{i}\left(\widetilde{\mathbf{x}}_{i}\right)$ depends only on the boundary geometry at the collocation point $\widetilde{\mathbf{x}}_{i}$.

The boundary is discretised into $N$ elements with $\Sigma=\bigcup_{j=1}^{N} \Sigma^{j}$, leading to a boundary approximation of the element field variables using the interpolation shape function $\phi$ of order $p$ :

$$
\begin{aligned}
& \sum_{i=1}^{p+1} \phi^{i} \widetilde{p}^{i}=\phi \widetilde{\mathbf{p}}^{e}, \\
& \sum_{i=1}^{p+1} \phi^{i} \widetilde{v}^{i}=\phi \widetilde{\mathbf{v}}^{e} .
\end{aligned}
$$

The proposed method employs the Lagrange interpolants relative to the Bernstein basis described in Section 2.2 as approximation shape functions. Thus, eqn (15) is written as:

$$
c_{i} \widetilde{p}_{i}=-\sum_{j=1}^{Q}\left[\iota \rho \omega\left\{\int_{\Sigma^{j}} \phi^{j} \widetilde{\Psi} d \Sigma\right\} \widetilde{v}^{j}+\left\{\int_{\Sigma^{j}} \phi^{j} \frac{\partial \widetilde{\Psi}}{\partial \mathbf{n}} d \Sigma\right\} \widetilde{p}^{j}\right],
$$

where $Q$ is the total number of nodes at the boundary and $\Sigma^{j}(\widetilde{\mathbf{x}})$ stands for the elements which contain the node $j$. The following integrals are defined for each collocation point $\widetilde{\mathbf{x}}_{i}$ and integration element $\Sigma^{j}$ :

$$
\begin{aligned}
& \mathbf{h}_{i}^{j}\left(\widetilde{\mathbf{x}}, \kappa_{z}, \omega ; \widetilde{\mathbf{x}}_{i}\right)=\int_{\Sigma^{j}} \phi^{j} \frac{\partial \widetilde{\Psi}}{\partial \mathbf{n}} d \Sigma, \\
& \mathbf{g}_{i}^{j}\left(\widetilde{\mathbf{x}}, \kappa_{z}, \omega ; \widetilde{\mathbf{x}}_{i}\right)=\int_{\Sigma^{j}} \phi^{j} \widetilde{\Psi} d \Sigma .
\end{aligned}
$$

Eqns (19) and (20) define the element matrices as in standard BEM formulation. Now, the Bézier-Bernstein space is used to describe the exact element geometry as $\Sigma^{j}(\widetilde{\mathbf{x}})=\mathbf{r}_{n}^{j}(t)$. Hence, the later integrals along a boundary element $\Sigma^{j}$ are rewritten in the univariate basis $t \in[0,1]$ as:

$$
\int_{\Sigma^{j}} \widetilde{f}\left(\widetilde{\mathbf{x}}, \kappa_{z}, \omega ; \widetilde{\mathbf{x}}_{i}\right) d \Sigma=\int_{0}^{1} f\left(\widetilde{\mathbf{x}}(t), \kappa_{z}, \omega ; \widetilde{\mathbf{x}}_{i}\right)\left|\frac{d}{d t} \mathbf{r}_{n}^{j}(t)\right| d t
$$


where $\tilde{f}\left(\widetilde{\mathbf{x}}, \kappa_{z}, \omega ; \widetilde{\mathbf{x}}_{i}\right)$ represents the kernels in eqns (19) and (20). Thus, eqn (21) is transformed into a symmetric integration interval $\xi \in[-1,1]$ to employ a Gauss-Legendre quadrature as:

$$
\int_{\Sigma^{j}} \tilde{f}\left(\widetilde{\mathbf{x}}, \kappa_{z}, \omega ; \widetilde{\mathbf{x}}_{i}\right) d \Sigma=\int_{-1}^{1} \tilde{f}\left(\widetilde{\mathbf{x}}(\xi), \kappa_{z}, \omega ; \widetilde{\mathbf{x}}_{i}\right)\left|\frac{d}{d t} \mathbf{r}_{n}^{j}(t)\right| \frac{d t(\xi)}{d \xi} d \xi .
$$

The relation $2 t=\xi+1$ defines the Jacobian of the transformation $d t / d \xi=1 / 2$.

Finally, eqns (19) and (20) are expressed as follows:

$$
\begin{aligned}
& \mathbf{h}_{i}^{j}\left(\widetilde{\mathbf{x}}, \kappa_{z}, \omega ; \widetilde{\mathbf{x}}_{i}\right)=\frac{1}{2} \int_{-1}^{1} \phi^{j} \frac{\partial \widetilde{\Psi}}{\partial \mathbf{n}}\left|\frac{d}{d t} \mathbf{r}_{n}^{j}(t)\right| d \xi \\
& \mathbf{g}_{i}^{j}\left(\widetilde{\mathbf{x}}, \kappa_{z}, \omega ; \widetilde{\mathbf{x}}_{i}\right)=\frac{1}{2} \int_{-1}^{1} \phi^{j} \widetilde{\Psi}\left|\frac{d}{d t} \mathbf{r}_{n}^{j}(t)\right| d \xi
\end{aligned}
$$

The system of equations for all the boundary elements becomes:

$$
\widetilde{\mathbf{H}}\left(\widetilde{\mathbf{x}}, \kappa_{z}, \omega ; \widetilde{\mathbf{x}}_{i}\right) \widetilde{p}\left(\widetilde{\mathbf{x}}, \kappa_{z}, \omega\right)=\widetilde{\mathbf{G}}\left(\widetilde{\mathbf{x}}, \kappa_{z}, \omega ; \widetilde{\mathbf{x}} i\right) \widetilde{v}\left(\widetilde{\mathbf{x}}, \kappa_{z}, \omega\right)
$$

where $\widetilde{\mathbf{H}}\left(\widetilde{\mathbf{x}}, \kappa_{z}, \omega ; \widetilde{\mathbf{x}}_{i}\right)$ and $\widetilde{\mathbf{G}}\left(\widetilde{\mathbf{x}}, \kappa_{z}, \omega ; \widetilde{\mathbf{x}}_{i}\right)$ are the fully non-symmetrical boundary element system matrices, and $\widetilde{p}\left(\widetilde{\mathbf{x}}, \kappa_{z}, \omega\right)$ and $\widetilde{v}\left(\widetilde{\mathbf{x}}, \kappa_{z}, \omega\right)$ are the sound pressure and particle normal velocity at the boundary $\Sigma$, respectively.

The boundary integrals are computed using a standard Gauss-Legendre quadrature with $4(p+1)$ integration points whenever the collocation point is sufficiently distant from the integration element. Otherwise, the solution of singular or weakly singular integrals is numerically computed using a smoothing transformation by means of a Gauss-Legendre quadrature [14]. Fig. 2 shows a scheme for the treatment of singular and weakly singular integrals. This figure represents a collocation point $\widetilde{\mathbf{x}}_{i}$ and an integration element $\Sigma^{j}$. The projection point $t\left(\widetilde{\mathbf{x}}_{i}\right)$ is found from $\widetilde{\mathbf{x}}_{i}$ as the point that minimizes the distance $r\left(\widetilde{\mathbf{x}}_{i}\right)$ to the integration element. Obviously, if the collocation point belongs to the integration element, then $t\left(\widetilde{\mathbf{x}}_{i}\right)$ coincides with the coordinate of this node. Once the point $t\left(\widetilde{\mathbf{x}}_{i}\right)$ has been identified, the element integration defined by eqn (21) becomes:

$$
\begin{aligned}
\int_{\Sigma^{j}} & \widetilde{f}\left(\widetilde{\mathbf{x}}, \kappa_{z}, \omega ; \widetilde{\mathbf{x}}_{i}\right) d \Sigma \\
\quad= & \int_{0}^{t\left(\widetilde{\mathbf{x}}_{i}\right)} f\left(\widetilde{\mathbf{x}}(t), \kappa_{z}, \omega ; \widetilde{\mathbf{x}}_{i}\right)\left|\frac{d}{d t} \mathbf{r}_{n}^{j}(t)\right| d t+\int_{t\left(\widetilde{\mathbf{x}}_{i}\right)}^{1} f\left(\widetilde{\mathbf{x}}(t), \kappa_{z}, \omega ; \widetilde{\mathbf{x}}_{i}\right)\left|\frac{d}{d t} \mathbf{r}_{n}^{j}(t)\right| d t,
\end{aligned}
$$

where the integration path is subdivided into two intervals according to $t\left(\widetilde{\mathbf{x}}_{i}\right)$. These integrals are numerically solved by a smoothing transformation of the form $\widetilde{\mathbf{x}}=\varphi_{l, r}(s)$ [14]:

$$
\varphi_{l, r}(s)=\frac{(\alpha+\beta-1) !}{(\alpha-1) !(\beta-1) !} \int_{0}^{s} u^{\alpha-1}(1-u)^{\beta-1} d u, \quad s \in[0,1]
$$

where

$$
\Xi_{\alpha, \beta}(s)=\int_{0}^{s} u^{\alpha-1}(1-u)^{\beta-1} d u
$$

is computed using the recurrence expression:

$$
\Xi_{\alpha, \beta}(s)=\frac{1}{\alpha+\beta-1}\left[-s^{\alpha-1}(1-s)^{\beta}+(\alpha-1) \Xi_{\alpha-1, \beta}(s)\right], \quad \alpha \geq 2, \beta \geq 1
$$




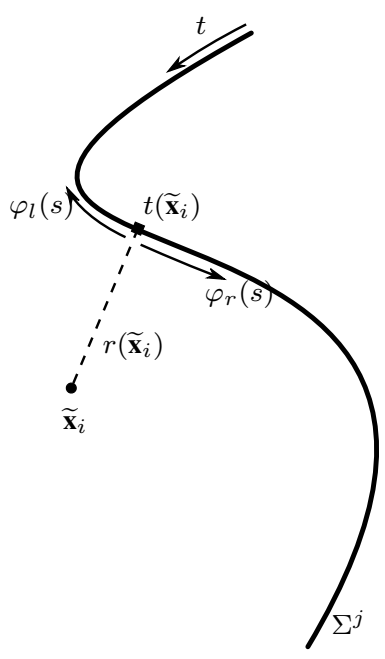

Figure 2: Treatment of singular and weakly singular integral.

with the initial value $\Xi_{1,1}(s)=s$. Accurate results for singular integrals are given in the next section, setting $\alpha=3$ and $\beta=1$, and using a number of integration points $10(p+1)$.

Therefore, eqn (26) becomes:

$$
\begin{aligned}
\int_{\Sigma^{j}} \widetilde{f}\left(\widetilde{\mathbf{x}}, \kappa_{z}, \omega ; \widetilde{\mathbf{x}}_{i}\right) d \Sigma= & -\int_{0}^{1} f\left(\widetilde{\mathbf{x}}\left(\varphi_{l}(s)\right), \kappa_{z}, \omega ; \widetilde{\mathbf{x}}_{i}\right)\left|\frac{d}{d t} \mathbf{r}_{n}^{j}(t)\right| \frac{d t}{d \varphi_{l}} \frac{d}{d s} \varphi_{l}(s) d s \\
& +\int_{0}^{1} f\left(\widetilde{\mathbf{x}}\left(\varphi_{r}(s)\right), \kappa_{z}, \omega ; \widetilde{\mathbf{x}}_{i}\right)\left|\frac{d}{d t} \mathbf{r}_{n}^{j}(t)\right| \frac{d t}{d \varphi_{r}} \frac{d}{d s} \varphi_{r}(s) d s
\end{aligned}
$$

where $d t / d \varphi_{l}=-t\left(\widetilde{\mathbf{x}}_{i}\right)$ and $d t / d \varphi_{r}=1-t\left(\widetilde{\mathbf{x}}_{i}\right)$.

The integration interval is then transformed to $\xi \in[-1,1]$ to employ a Gauss-Legendre quadrature as described for regular integrals. The two integrals are reduced to only one integral if the projection point $t\left(\widetilde{\mathbf{x}}_{i}\right)$ lies at the element endpoints.

We chose a maximum radius $r^{*}$ between the collocation point and the integration element for the identification of singular integrals. Otherwise, the collocation point is sufficiently distant from the integration element and the resulting integrals are regular. The asymptotic behaviour of the fundamental solution is accounted for by selecting the critical radius $r^{*}$ when the integral becomes singular [15]:

$$
\begin{aligned}
& \lim _{r \rightarrow 0} \widetilde{\Psi}=\frac{\iota}{4}+\frac{1}{2 \pi} \log \left(\kappa_{\alpha} r\right), \\
& \lim _{r \rightarrow 0} \frac{\partial \widetilde{\Psi}}{\partial n}=\frac{1}{2 \pi r} \frac{\partial r}{\partial n} .
\end{aligned}
$$

\section{NUMERICAL EXAMPLE}

In this section, the capability of the proposed method is shown with a numerical example. The problem solved herein models the three-dimensional wave propagation in a fluid channel with a fixed scatterer. The fluid channel is bounded by two reflection planes that were assumed to 
be rigid with null particle normal velocity. The total channel height was $H=6 \mathrm{~m}$ and fluid air properties were taken with sound wave propagation velocity $c_{f}=340 \mathrm{~m} / \mathrm{s}$ and density $\rho=1.225 \mathrm{~kg} / \mathrm{m}^{3}$.

A modified Green's function for the fluid channel was used to limit the discretisation to the scatterer boundary. The method of images was used to construct the modified Green's function [15]. The scatterer and the fluid channel were centred at the origin. The fluid channel and the scatterer were excited by a point source acting at location $\mathbf{x}=(-3,0,0) \mathrm{m}$ with $\kappa=20 \mathrm{rad} / \mathrm{m}$. The source was defined by the modified Green's function. The problem solution was computed in the frequency-wavenumber domain for a longitudinal wavenumber varying over a range from zero to $\kappa_{z}=2 \kappa$ in 128 equidistant steps. The pressure field was evaluated over a grid of receivers with a density of six points per wavelength.

Two geometries were considered: (i) a cylindrical scatterer and (ii) the complex shaped scatterer represented in Fig. 3(a) directly imported from a CAD model. The geometry of the second example was defined by twelve $C^{1}$ cubic patches. Problem discretisation was given by $\kappa h=6$ and $d_{\lambda}=6$, which leads to an element order $p=6$. The element nodes were located at the Chebyshev points of the second kind. Once the boundary discretisation had been defined, the geometry patches were subdivided as shown in Fig. 3(b). The cylindrical scatterer was discretised following a similar procedure.

Fig. 4 shows the incident wavefield and the scattered wave. The representation is limited to a half model, according to the problem symmetry. The incident pressure field shows a spherical distribution that was gradually lost as the distance to the source point increased due to channel wave refraction (Fig. 4(a)). The amplitude decreased with the distance to the excitation point, according to the radiation condition. The pattern of the incident wave changed under the effect of the scatterers. A complex pattern was found, caused by waves reflected from both the channel borders and the scatterer boundary. A shadow region was found behind the scatterers where pressure amplitude was considerably lower.

This example has shown that the proposed method is, therefore, a useful tool for studying boundary problems with exact CAD geometry. An independent geometry and variable field approximation allowed efficient modelling that took both the complexity of the boundary shape and the studied wavenumber into account. In this case, it was enough to use cubic

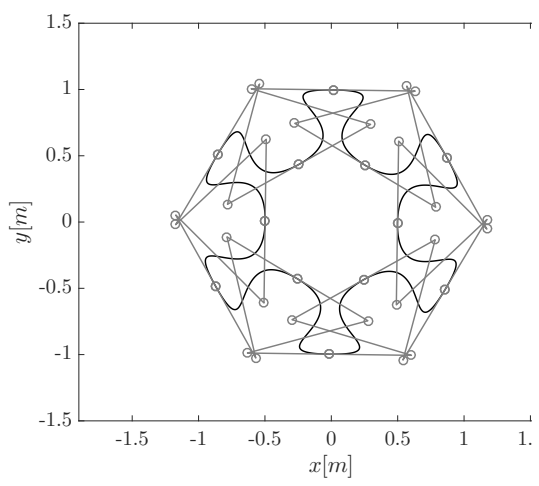

(a)

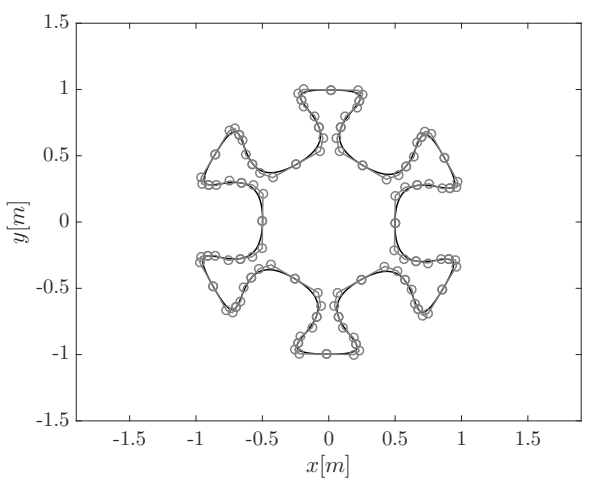

(b)

Figure 3: (a) Complex geometry imported from CAD model; (b) Related discretisation for $\kappa h=6$. Control points and their related control polygons are represented by grey circles and grey lines. 


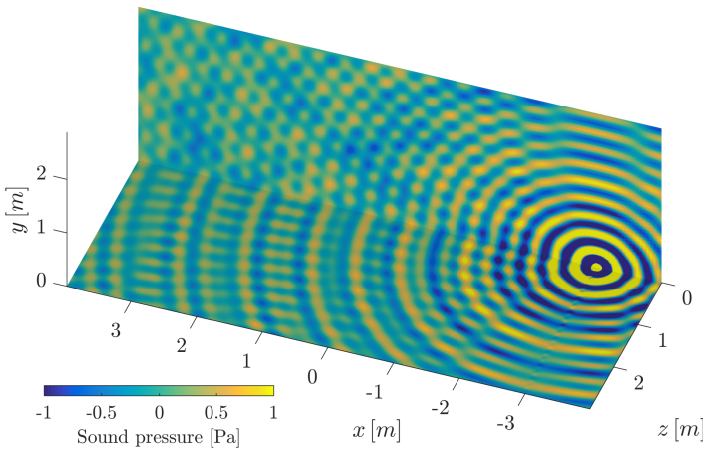

(a)

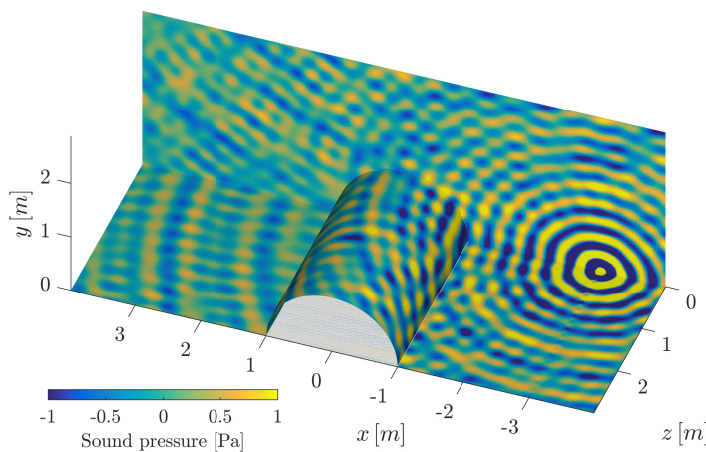

(b)

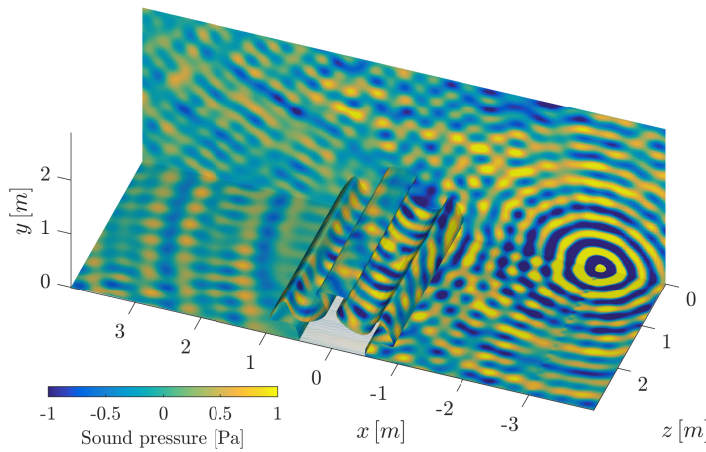

(c)

Figure 4: Real part of (a) incident wavefield, and the scattered wavefield by (b) a cylindrical cavity, and (c) a complex shape boundary. 
curves for the geometry approximation, while it was necessary to use six order elements for the variable field.

\section{CONCLUSIONS}

This paper has proposed a novel formulation of the BEM to consider exact CAD geometry. The boundary geometry is described in the Bézier-Bernstein space which is commonly used in computer-aided design. Boundary elements are defined by the subdivision of Bézier curves according to an $h$-refinement strategy. The element geometry is evaluated and subdivided using the de Casteljau algorithm and the polar form of Bernstein polynomials. Geometric operations are easily performed without CAD interaction which entails a great flexibility for $h$-refinement.

The field variables are approximated independently of the geometry representation. Therefore, the proposed method is arbitrarily defined as subparametric, isoparametric or superparametric, depending on the problem properties and boundary geometry. The approximation of field variables has been generalised considering different shape functions families defined from node locations and element degree, using the Newton-Bernstein algorithm.

The boundary element integrals are solved according to distance from collocation points and the integration element. The asymptotic behaviour of the fundamental solution has given a critical radius below which the element integrals become singular. In this case, singular integrals have been numerically integrated with a polynomial transformation of the integration domain.

In particular, this work has been used to solve the Helmholtz equation in longitudinally invariant domains, but it is also possible to apply it in different two-dimensional or $2.5 \mathrm{D}$ physical problems, using an appropriate fundamental solution for the boundary element method.

\section{ACKNOWLEDGEMENTS}

The research work presented herein was supported by the Spanish Ministry of the Economy and Competitiveness (Ministerio de Economía y Competitividad) through research project BIA2016-75042-C2-1-R. It was also supported by project POCI-01-0247-FEDER-017759, funded by Portugal 2020 through the Operational Programme for Competitiveness Factors (COMPETE 2020). Financial support is gratefully acknowledged. The authors also wish to acknowledge the support provided by the Andalusian Scientific Computing Centre (CICA).

\section{REFERENCES}

[1] Atroshchenko, E. \& Bordas, S.P.A., Geometry-independent field approximation for spline-based finite element methods. 11th World Congress on Computational Mechanics, 2014.

[2] Farouki, R.T., The Bernstein polynomial basis: A centennial retrospective. Computer Aided Geometric Design, 29(6), pp. 379-419, 2012.

[3] Farouki, R.T. \& Rajan, V.T., Algorithms for polynomials in Bernstein form. Computer Aided Geometric Design, 5(1), pp. 1-26, 1988.

[4] Farouki, R.T., Legendre-Bernstein basis transformations. Journal of Computational and Applied Mathematics, 119(1-2), pp. 145-160, 2000.

[5] Bouchon, M. \& Aki, K., Discrete wave-number representation of seismic source wavefields. Bulletin of the Seismological Society of America, 67(2), pp. 259-277, 1977. 
[6] Romero, A., Tadeu, A., Galvín, P. \& António, J., 2.5D coupled BEM-FEM used to model fluid and solid scatterering wave. International Journal for Numerical Methods in Engineering, 101(2), pp. 148-164, 2015.

[7] Ramshaw, L., Blossoming: A connect-the-dots approach to splines. Digital Equipment Corporation SRC Report No. 19.

[8] Seidel, H.-P., An Introduction to Polar Forms. IEEE Compututer Graphics and Applications, 13(1), pp. 38-46, 1993.

[9] Dokken, T., Dæhlen, M., Lyche, T. \& Mørken, K., Good approximation of circles by curvature-continuous Bézier curves. Computer Aided Geometric Design, 7(1-4), pp. 33-41, 1990.

[10] Hur, S. \& Kim, T.-W., The best G1 cubic and G2 quartic Bézier approximations of circular arcs. Journal of Computational and Applied Mathematics, 236(6), pp. 1183$1192,2011$.

[11] Ainsworth, M. \& Sánchez, M.A., Computing the Bézier control points of the lagrangian interpolant in arbitrary dimension. SIAM Journal on Scientific Computing, 38(3), pp. A1682-A1700, 2016.

[12] Farouki, R.T., Goodman, T.N.T. \& Sauer, T., Construction of orthogonal bases for polynomials in Bernstein form on triangular and simplex domains. Computer Aided Geometric Design, 20(4), pp. 209-230, 2003.

[13] Romero, A., Galvín, P., António, J., Domínguez, J. \& Tadeu, A., Modelling of acoustic and elastic wave propagation from underground structures using a 2.5D BEM-FEM approach. Engineering Analysis with Boundary Elements, 76, pp. 26-39, 2017.

[14] Monegato, G. \& Scuderi, L., Numerical integration of functions with boundary singularities. Journal of Computational and Applied Mathematics, 112(1-2), pp. 201214, 1999.

[15] Wu, T., Boundary Element Acoustics Fundamentals and Computer Codes, Advances in Boundary Elements, WIT Press, 2000. 